\title{
Presence of new mutations in the TP53 gene in patients with low-risk myelodysplastic syndrome: two case reports
}

\author{
Fernando Barroso Duarte ${ }^{1}$, Romélia Pinheiro Gonçalves Lemes ${ }^{2}$, Talyta Ellen de Jesus dos Santos ${ }^{2 *}$, \\ Maritza Cavalcante Barbosa ${ }^{2}$, João Paulo Leitão de Vasconcelos ${ }^{1}$, Francisco Dário Rocha-Filho ${ }^{1}$, Ilana Zalcberg ${ }^{3}$, \\ Diego Coutinho ${ }^{3}$, Monalisa Feliciano Figueiredo ${ }^{2}$, Luciana Barros Carlos ${ }^{4}$ and Paulo Roberto Leitão de Vasconcelos ${ }^{1}$
}

\begin{abstract}
Background: Myelodysplastic syndromes are heterogeneous disorders. Patients with myelodysplastic syndrome disease often have ineffective hematopoiesis, cytopenias, blood cell dysplasia in one or more cell types, and are at high risk for developing acute myeloid leukemia. In myelodysplastic syndrome, mutations of TP53 gene are usually associated with complex karyotype and confer a worse prognosis. In the present study, two mutations in this gene are presented and discussed with the clinical evolution of the patients.

Case presentation: The first case is a 77-year-old Brazilian woman diagnosed as having multiple lineage dysplasia myelodysplastic syndrome according to World Health Organization 2016 and classified as very low-risk by Revised International Prognostic Scoring. The second case is an 80-year-old Brazilian man also diagnosed as having multiple lineage dysplasia myelodysplastic syndrome and classified as low risk. The mutation described in the first case was already identified in some neoplasias and it is associated with a poor prognosis, but it had never been reported before in myelodysplastic syndrome. The second mutation has never been described.

Conclusions: This is a novel report for the scientific community and may be very helpful as we can better understand the disease and the impact of mutations through the follow-up of these patients and others in the future. Both patients are in a good clinical condition, suggesting that these mutations may not alter the clinical course of the disease or may be associated with a good prognosis, but their role in the disease must be investigated more deeply in a larger population.
\end{abstract}

Keywords: Myelodysplastic syndromes, TP53 mutations, Prognosis

\section{Background}

Myelodysplastic syndromes (MDS) are heterogeneous disorders. The TP53 gene is implicated in the manifestation of hematologic features, specifically macrocytic anemia, frequently involved in MDS. Mutations in the TP53 gene are usually associated with complex karyotype and confer a worse prognosis. Somatic mutations in the TP53 tumor suppressor gene are found in approximately $50 \%$ of all human tumors, making it the most commonly mutated gene. The expression of p53 protein

\footnotetext{
* Correspondence: talytaellen08@hotmail.com

${ }^{2}$ Research Laboratory in Hemoglobinopathies and Genetics of Hematologic Diseases, Federal University of Ceará, Capitão Francisco Pedro street, n. 1210, Rodolfo Teófilo, Fortaleza, Ceara, Brazil

Full list of author information is available at the end of the article
}

and the study of mutations are important tools, mainly for the prognosis of MDS [1].

Although the biological and clinical roles that a normal and altered p53 protein play in cancer remain areas of intense investigation and debate, a number of studies have shown that alterations in p53 are either associated or not with patient outcomes, such as response to therapy or survival [2].

In this context, we present two case reports of very low-risk and low-risk MDS, according to the Revised International Prognostic Scoring System (IPSS-R), with two TP53 gene mutations described for the first time in patients with MDS and we describe the outcomes of these patients. 
Table 1 Clinical and hematological features of both patients

\begin{tabular}{lll}
\hline & Mutation & \\
\cline { 2 - 3 } & C.394A $>T$ & c.783-1_784delGTG \\
\hline Age (years) & 79 & 82 \\
Gender & Female & Male \\
Complete blood count & & \\
Hemoglobin, $\mathrm{g} / \mathrm{dL}$ & 10.3 & 10.82 \\
Hematocrit, $\%$ & 30.0 & 33.0 \\
Mean corpuscular volume $(\mathrm{fL})$ & 88.08 & 92.5 \\
Leukocytes $\left(\times 10^{9} / \mathrm{L}\right)$ & 5.5 & 4.59 \\
Neutrophils $\left(\times 10^{9} / \mathrm{L}\right)$ & 3.3 & 0.77 \\
Platelets $\left(\times 10^{9} / \mathrm{L}\right)$ & 208 & 113.2 \\
Karyotype & $46, X X$ & $46, X Y$ \\
Bone marrow blasts & $0.5 \%$ & $2.2 \%$ \\
Bone marrow fibrosis & Degree $\|$ & Without fibrosis \\
Bone marrow iron & Normal & Normal \\
Bone marrow cellularity & Hypocellular & Hypercellular \\
CD34 immunoreactivity & Negative & Negative \\
p53 immunohistochemistry & Negative & Positive (2\%) \\
Transfusion dependence & No & No \\
\hline
\end{tabular}

Both patients are enrolled in a cross-sectional analytical study with adult patients of both genders diagnosed with low-risk MDS, receiving out-patient treatment at Walter Cantídio University Hospital. The differential diagnosis was performed in all patients. Peripheral blood samples were collected with ethylenediaminetetraacetic acid (EDTA) for laboratory analysis.

Initially, p53 protein expression of 38 patients with low-risk MDS was determined by immunohistochemistry $[3,4]$. At a second stage, 37 patients were evaluated for mutations in the TP53 gene by Sanger sequencing. Among these, only two patients had mutations. The study was approved by Walter Cantídio University Hospital Ethics Committee. Informed consent was obtained from all patients. Other causes of clonal or nonclonal cytopenias were excluded. Common etiologies of cytopenias or morphological abnormalities that may mimic MDS, such as aplastic anemia or paroxysmal nocturnal hemoglobinuria were investigated.
Molecular analysis of the TP53 gene was performed at the Laboratory of Molecular Biology of the Bone Marrow Transplant Center (CEMO), Cancer Institute (INCA) in Rio de Janeiro, by direct sequencing (Sanger sequencing). Four pairs of primers were used for complete coverage of exons: 3-9; primer exon 3-4, primer exon 5-6, primer exon 7, and primer exon 8-9.

Exons 3-9 of the gene were amplified by polymerase chain reaction (PCR) in deoxyribonucleic acid (DNA) extracted from leukocytes. The PCR primers and conditions for amplification of genomic DNA followed those established by the International Agency for Research on Cancer (p53.iarc.fr/ProtocolsAndTools.aspx). All PCR products were confirmed by $1.5 \%$ agarose gel, purified using Wizard SV Gel kits and PCR Clean-Up (both by Promega) and sequenced in a 16-capillary automated sequencer (ABI PRISM ${ }^{\circ} 3100$ Genetic Analyzer, Applied Biosystems). The sequence data files were analyzed using Mutation Surveyor (SoftGenetics) software. All variants found were compared with the databases: COSMIC, $\mathrm{dbSNP}$, and 1000 genomes UniProtKB.

\section{Case presentation \\ Case report 1}

In 2011, a 77-year-old Brazilian woman was admitted to our service due to refractory anemia. She was treated with vitamin B12, folic acid, and iron, but she showed no improvement. A bone marrow aspirate (5 January 2011) showed dyserythropoiesis greater than $20 \%$, dysgranulopoiesis greater than $20 \%$, and $4 \%$ of blasts; her iron level was normal. A second bone marrow aspirate (8 June 2011) showed hypocellularity with dyserythropoiesis and dysgranulopoiesis (less than 10\%), $1 \%$ of blasts, and absent iron. A third myelogram (18 August 2013) showed mild dysmyelopoiesis, hypocellularity with $7 \%$ dysmegakaryopoiesis, and moderate dysgranulopoiesis (12\%); there was also a reduced deposit of iron and $0.5 \%$ of blasts (Fig. 1). The first bone marrow biopsy (8 June 2011) showed hypocellularity, $20 \%$ of dyserythropoiesis, abnormal localization of immature myeloid precursors (ALIPs), and dysplastic megakaryocytes. The second bone marrow biopsy (12 August 2013) showed hypocellular bone marrow with dysmegakaryopoiesis and dyserythropoiesis associated

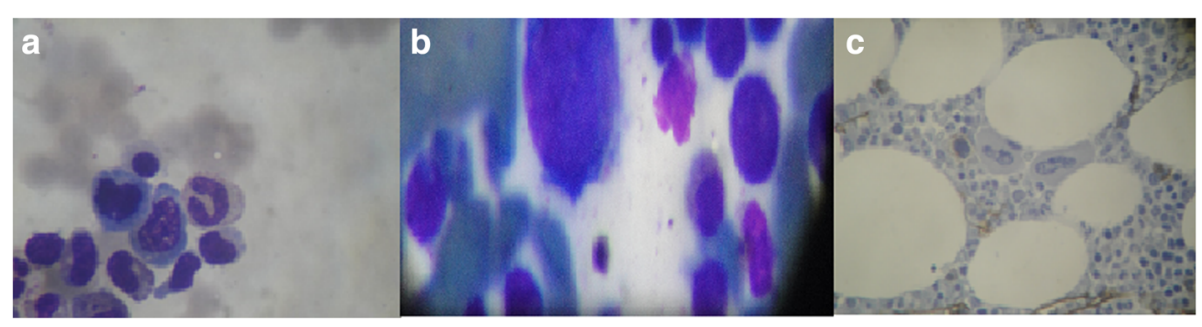

Fig. 1 Myelogram of patient 1. a Dyserythropoiesis; b dysmegakaryopoiesis; c reticulin stain 


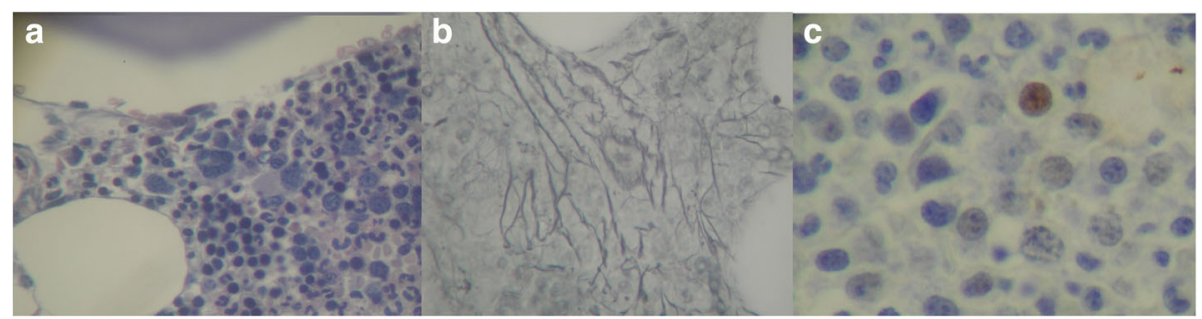

Fig. 2 a Myelogram of patient 2. a Hypercellularity; b mild focal thickening with reticulin staining; c p53 expression by immunohistochemistry at 2\%

with myelofibrosis degree II. The immunohistochemical studies of p53 expression and anti-CD34. ${ }^{+}$were negative. Serology for hepatitis B, hepatitis C, human immunodeficiency virus (HIV), and human T-cell lymphotropic virus (HTLV) were negative. Her thyroid hormones, ferritin, and dehydrogenase lactate levels were normal. A differential diagnosis for aplastic anemia was performed. Cytogenetic analysis showed a normal female karyotype. She was diagnosed as having multiple lineage dysplasia MDS, according to World Health Organization (WHO) 2016 and classified as very low risk based on IPSS-R (score-1). She currently shows no clinical or laboratory manifestations of the disease (Table 1).

\section{Case report 2}

In 2011, an 80-year-old Brazilian man, presented to our hospital with anemia, thrombocytopenia, and monocytosis $\left(>1000 / \mathrm{mm}^{3}\right)$. He had dizziness and normal blood pressure levels. A myelogram showed dyserythropoiesis, dysgranulopoiesis, and dysmegakaryopoiesis; blasts were approximately $2.2 \%$ (Fig. 2). Cytogenetic analysis showed a normal karyotype. A bone marrow biopsy showed hypercellularity with significant expansion of granulocytic and megakaryocytic series. Mild focal reticulin thickening was observed. He had not received any blood transfusions and had no infection reports. Routine examinations, including renal function and electrolytes, were within the normal ranges. An immunohistochemistry analysis showed normal expression of precursor cells; there was a moderate increase in vasculature and nuclear positive reaction to p53 (2\%). The diagnosis was MDS with multiple lineage dysplasia. He was classified as low-risk MDS, according to IPSS-R (score2.2 (Table 1)).

Currently, he is 85 and has difficulty walking, but with a good overall health status, despite his limitations. In both cases, patients with these mutations have had a good evolution despite a positive p53 expression.

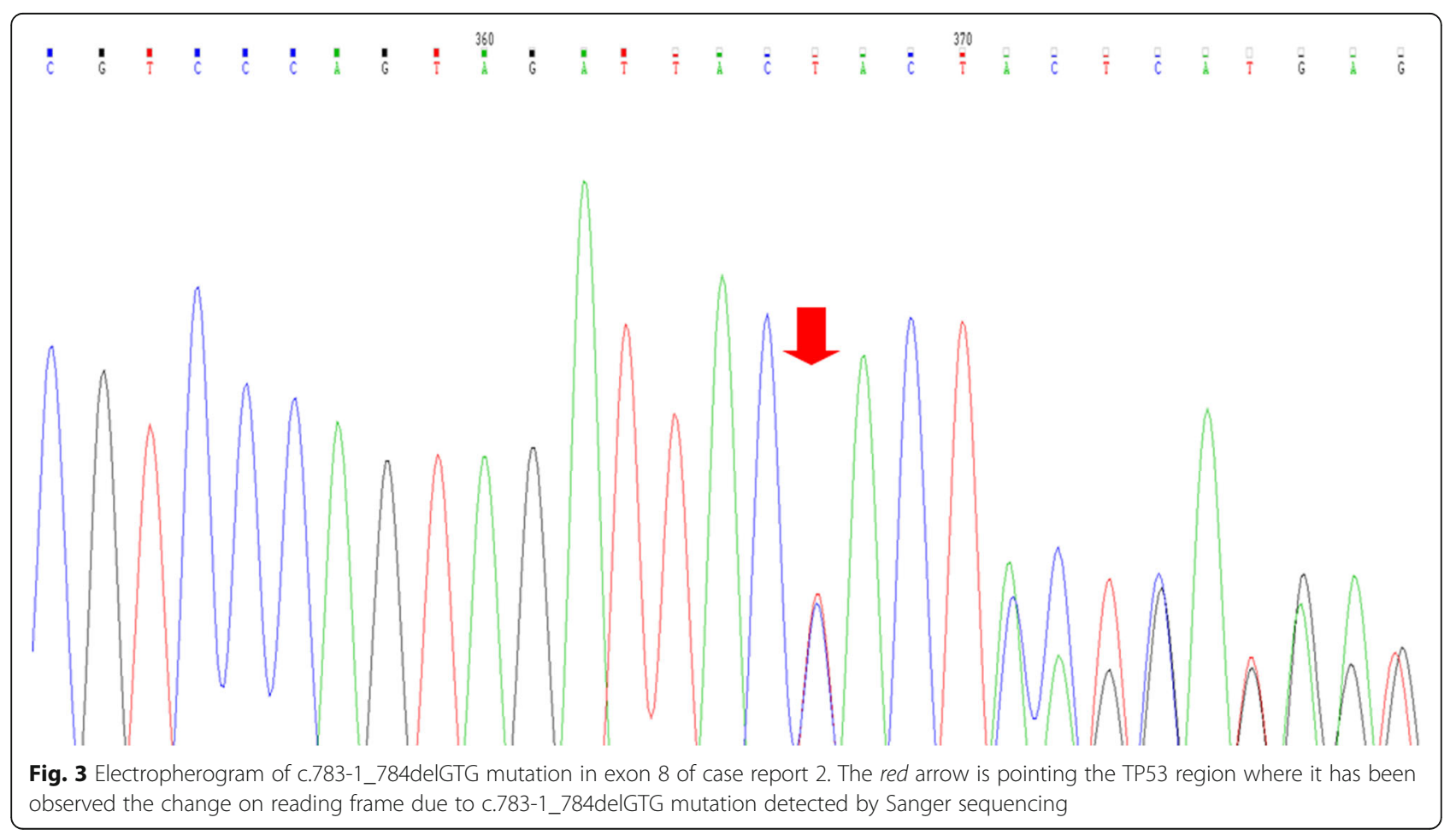




\section{Discussion}

P53 protein is a key regulator of stem cell homeostasis that impacts on the array of cellular functions including genomic surveillance, cell cycle regulation, and apoptotic and inflammatory response [5].

Inactivation of p53 has been reported in hematologic malignancies in association with disease progression. In adult patients with de novo MDS or acute myeloid leukemia (AML), somatically acquired TP53 mutations are observed in approximately $10 \%$ of cases, and are often associated with loss of the short arm of chromosome 17, a complex karyotype, resistance to chemotherapy, and a short survival $[2,6]$.

In our case reports, the patients were low risk and very low risk and both had a normal karyotype. Case 1 exhibited c.394A $>\mathrm{T}$ (p.K132\%) nonsense mutation, which generates a non-functional protein [7]. Case 2 had a novel TP53 c.783-1_784delGTG mutation (Fig. 3).

P53 normally interacts with a variety of proteins involved in transcriptional regulation, DNA repair, cell cycle progression, apoptosis, and proteasome-mediated protein degradation. Although the biological and clinical roles that normal and altered p53 play in cancer remain areas of intense investigation and debate, a number of studies have shown that alterations in p53 are either associated or not with patient outcomes, such as response to therapy or survival $[8,9]$.

In both cases, mutations were found in low-risk MDS. Considering the impact of TP53 gene on hematologic malignancies, these two mutations may or may not contribute to the knowledge of the role of TP53 in disease pathogenesis, tumor progression, and to the identification of new therapeutic targets.

\section{Conclusions}

The present study discloses a surprising outcome to the scientific community. The TP53 c.783-1_784delGTG mutation has been identified for the first time. Regarding the other mutation, p.K132\%, it has been considered in other types of tumors as a poor prognostic factor; in this specific case, it does not seem to be associated with complications regarding the patient's clinical condition. This is a relevant result and more studies are needed to evaluate the role of these mutations in MDS.

\section{Acknowledgements}

The authors would like to thank the patients for their collaboration, the staff of the Hematology service from University Hospital Walter Cantídio, and the academic researchers.

\section{Funding}

Not applicable.

\section{Availability of data and materials}

Data sharing is not applicable to this article as no datasets were generated or analyzed during the current study.

\section{Authors' contributions}

FDR, RPGL PRLV, and LBC participated in the design and coordination of the study and helped to draft the manuscript. FDR and JPLV conceived of the study and participated in the design of the study. FDRF conducted medical examinations and image acquisition. IZ and DC carried out the molecular genetic studies. TEJS, MCB, and MFF participated in the sequence alignment and drafted the manuscript. All authors read and approved the final manuscript

\section{Competing interests}

The authors declare that they have no competing interests.

\section{Consent for publication}

Written informed consent was obtained from the patients for publication of this case report and any accompanying images. A copy of the written consent is available for review by the Editor-in-Chief of this journal.

\section{Ethics approval and consent to participate}

The study was approved by the Ethics Committee of the Federal University of Ceará with protocol number 129/12, and carried out in accordance with the Declaration of Helsinki for experiments involving humans. Informed consent was obtained from all patients.

\section{Publisher's Note}

Springer Nature remains neutral with regard to jurisdictional claims in published maps and institutional affiliations.

\section{Author details}

${ }^{1}$ Department of Surgery, Federal University of Ceará, Fortaleza, Ceara, Brazil. ${ }^{2}$ Research Laboratory in Hemoglobinopathies and Genetics of Hematologic Diseases, Federal University of Ceará, Capitão Francisco Pedro street, n. 1210, Rodolfo Teófilo, Fortaleza, Ceara, Brazil. ${ }^{3}$ Laboratory of Molecular Biology-Center for Bone Marrow Transplantation, CEMO-National Cancer Institute - INCA, Rio de Janeiro-Rio de Janeiro, Brazil. ${ }^{4}$ Center of Hematology and Hemotherapy of Ceara - Cryobiology Laboratory, Fortaleza, Ceara, Brazil.

Received: 22 September 2016 Accepted: 29 March 2017

Published online: 21 May 2017

\section{References}

1. Jhanwar SC. Genetic and epigenetic pathways in myelodysplastic syndromes: A brief overview. Adv Biol Regul. 2015;58:28-37.

2. Haferlach C, Dicker F, Herholz H, Schnittger S, Kern W, Haferlach T. Mutations of the TP53 gene in acute myeloid leukemia are strongly associated with a complex aberrant karyotype. Leukemia. 2008;22:1539-41.

3. Reis SC, Traina F, Metze K, Saad ST, Lorand-Metze I. Variation of bone marrow CD34+ cell subsets in myelodysplastic syndromes according to who types. Neoplasma. 2009;56(5):435-40.

4. Della Porta MG, Malcovati L, Boveri E, Travaglino E, Pietra D, Pascutto C, Passamonti F, Invernizzi R, Castello A, Magrini U, Lazzarino M, Cazzola M. Clinical relevance of bone marrow fibrosis and CD34-positive cell clusters in primary myelodysplastic syndromes. J Clin Oncol. 2009;27(5):754-62.

5. McGraw KL, Zhang LM, Rollison DE, Basiorka AA, Fulp W, Rawal B, Jerez A, Billingsley DL, Lin HY, Kurtin SE, Yoder S, Zhang Y, Guinta K, Mallo M, Solé F, Calasanz MJ, Cervera J, Such E, González T, Nevill TJ, Haferlach T, Smith AE, Kulasekararaj A, Mufti G, Karsan A, Maciejewski JP, Sokol L, Epling-Burnette PK, Wei S, List AF. The relationship of TP53 R72P polymorphism to disease outcome and TP53 mutation in myelodysplastic syndromes. Blood Cancer J. 2015;5, e291.

6. Seifert H, Mohr B, Thiede C, Oelschlägel U, Schäkel U, Illmer T, Study Allianc e Leukemia (SAL), et al. The prognostic impact of 17p (p53) deletion in 2272 adults with acute myeloid leukemia. Leukemia. 2009;23:656-63.

7. Catalogue of somatic mutations in cancer. http://cancer.sanger.ac.uk/ cosmic/mutation/overview?id=44641. Accessed 28 May 2015.

8. Shahin MS, Hughes JH, Sood AK, et al. The prognostic significance of p53 tumor suppressor gene alterations in ovarian carcinoma. Cancer. 2000;89: 2006-17.

9. Reles A, Wen WH, Schmider A, et al. Correlation of p53 mutations with resistance to platinum-based chemotherapy and shortened survival in ovarian cancer. Clin Cancer Res. 2001;7:2984-97. 